\title{
Robotic Systems Involved in the Diagnosis of Neurodegenerative Diseases
}

\author{
Anastasios Xiarchos
}

The continuing development of robotics on the one hand and, on the other hand, the estimated relative growth in the number of elderly individuals suffering from neurodegenerative diseases raises the question of which contribution these powerful systems could have to assist or even improve the diagnostic procedure. Up to now many research groups have designed protocols that use robotic systems that measure the performance of the subject through tracking tasks with and without a synchronized cognitive or motor task. Also, new robotic noninvasive technologies are designed for the diagnosis of neurodegenerative diseases, through the analysis of eye movements. These assessments can distinguish individuals with the disease from age-matched controls. The objective of this review is to evaluate the effects and efficiency of robot interventions in the diagnosis of these devastating neurological diseases.

\footnotetext{
A. Xiarchos ( $\square)$

Medical Centre of Athens, Athens, Greece

e-mail: info@axiarchos.gr 